\title{
Acaricidal Property of Certain Indigenous Herbal Extracts against Citrus Leaf Mite, Panonychus citri on Acid Lime
}

\author{
C. Chinniah, K. Naveena* and M. Shanthi \\ Department of Agricultural Entomology, Agricultural College and Research Institute \\ Tamil Nadu Agricultural University, Madurai - 625 104, Tamil Nadu, India \\ *Corresponding author
}

\section{A B S T R A C T}

\section{Keywords}

Citrusleaf mite,

Panonychus citri,

Indigenous herbal

pesticides, Acid

lime

Article Info

Accepted:

18 April 2020

Available Online:

10 May 2020
The field investigation was carried out to evaluate the acaricidal property of certain very common indigenous and endemic medicinal herbs in field conditions against citrus leaf mite, $P$. citri at Madurai Agricultural College and Research Institute during summer 2018. Among the herbal extracts evaluated, Citrulus fruit extract registered 74.90 per cent of mite reduction which was closely followed by basil leaf extract (68.12\% reduction) and notchi leaf extract (61.75\% reduction) over untreated check, coupled with higher fruit yield $(9.60 \mathrm{t} / \mathrm{ha}, 8.50 \mathrm{t} / \mathrm{ha}, 8.10 \mathrm{t} / \mathrm{ha})$ and increased cost benefit ratio $(1: 2.2,1: 2.0,1: 1.9)$, respectively.

\section{Introduction}

Citrus red mite (P. citri) is one of the key pests of citrus and distributed all over the world where citrus is cultivated predominantly. Nymphs and adults sucks the nutrients from the leaf and fruits by means of their long stylets. The feeding produces tiny grey or silvery spots on leaves and fruits known as stippling. Damage to leaves inhibits photosynthesis, increases transpiration which may lead to necrosis. Severe infestations can lead to premature leaf fall, discoloration of fruits, dieback symptom and decreased plant vigour (Kranz, 1977). Citrus is commercially grown in the southern regions of Tamil Nadu.

India which ranks sixth among the citrus growing countries in the world. The citrus cultivation ranks third in an area, next to mango and banana in India. Citrus orchards occupy an area of 10.55 lakh hectares in the country with an annual production of 127.46 lakh tonnes of fruits with the productivity of 9.90 tonnes per hectare (Poovizhiraja et al., 2019). 
Around 823 species of insects and mite pests are known to devastate citrus crop in various stages. More than 30 per cent of citrus production is lost in India every year due to the menace by more than 165 species of mites and insect pests. Many of these pests damages the crop at all stages of crop growth (Butani, 1979). More than 25 species of phytophagous mites alone have been reported to infest citrus crop in India (Prasad, 1974; Dhooria and Gupta, 1998).

Losses due to injury by mites are alarming when climatic conditions are more favourable for their multiplication. LeClerg (1965) recorded $2.5 \%$ loss in the production of citrus fruits due to the spider mite damage alone in USA. Seven species of phytophagous mites namely spider mites (Eutetranychus orientalis, P. citri, Schizotetranychus hindustanicus), false spider mites (Brevipalpus phoenicis, B. californicus), tarsonemid mites (Polyphagotarsonemus latus) and eriophyid mites (Eriophyes sheldoni, Phyllocoptruta oleivora) have been reported as serious pests of citrus from different parts of India (Dhooria et al., 2005).

Since many years, the management of insect and mite pests could be done through chemical insecticides and acaricides by farmers with the aim of higher benefits. The indiscriminate use of insecticides caused adverse effects, like resurgence, outbreak of minor pests besides residues in fruits which obviously resulting in increased cost of cultivation.

The replacement of synthetic pesticides with natural plant extracts could preserves natural enemies and reduces environmental risks (Rao et al., 2015). Keeping these points in view, the present study was aimed to evaluate the effect of different indigenous herbal extracts against citrus leaf mite in an ecofriendly manner.

\section{Materials and Methods}

The field experiment was conducted to assess the acaricidal propertiesof certain very common and endemic herbal extracts against citrus leaf mite, $P$. citri in a farmer's holding at Azhagar kovil village of Melur block, Madurai during summer 2018. The field experiment was laid out in a Randomized block design with three replications per treatment. Each tree was considered as a replication and the mite population was assessed from twenty leaves per plant drawn at random and the mean population per twenty leaves was worked out after each spray.

Totally three sprays were applied at 15 days interval and the population of test mite was assessed on 1,3,7 and 14 days after each spray apart from pre-treatment count to ensure that the initial population was above the threshold level. The data on mite population were subjected to square root transformation followed by LSD to group the treatments according to their efficacy. The fruit yield per tree was recorded as and when harvested and computed in terms of tons/ ha to work out the incremental cost benefit ratio, taking into account the current market price of fruits. The ethanolic extracts of plant tissues were prepared by Soxhlet apparatus and the required concentrations were prepared for spraying. Teepol@1\% was added to the spray fluid for better stickyness.

\section{Results and Discussion}

The field count on the mean number of mites per leaf recorded after I spray, suggested that the standard check propargite @ 50 EC 2 ml/ lit. recorded minimum number (3.4 No./ leaf) followed by Citrulus fruit extract (6.4 No./ leaf) which was statistically on par with basil leaf extract (7.7 No./ leaf) and vitex leaf extract (8.7 No./ leaf) (Table 1). 
Almost similar trend was recorded at the second round after spray with reference to mite reduction nevertheless the standard check propargite @ 50 EC $2 \mathrm{ml} /$ lit. recorded minimum number of mites (5.0 No./ leaf) which was statistically on par with Citrulus fruit extract (6.5 No./ leaf) followed by basil leaf extract (8.6 No./ leaf) (Table 2). The field data on mite population per leaf recorded after III spray, also suggested that the standard check propargite 50 EC @ $2 \mathrm{ml} /$ lit. recorded minimum number of mites (5.2/ leaf) which was statistically on par with Citrulus fruit extract (6.0 No./ leaf) followed by basil leaf extract (7.8 No./ leaf) (Table 3 ).

The comparative check propargite 50 EC recorded the highest per cent reduction of mite over untreated check $(82.07 \%)$ followed by Citrulus fruit extract(74.90\%), basil leaf extract $(68.12 \%)$ and vitex leaf extract $(61.75$ $\%)$ respectively. Similarly, this has reflected on the incremental yield and cost benefit ratio also.

The increased yield was recorded in case of propargite 50 EC @ 2 ml/ lit. (11.20 t/ha) followed by Citrulus fruit extract @ 5\% (9.60 t/ha), basil leaf extract @ 5\% (8.50 t/ha) and vitex leaf extract@ @\% (8.10 t/ha) and. The sweet flag rhizome extract and adathoda leaf extract@5\% recorded the moderate to low per cent reduction of 53.38 and 50.19 per cent respectively (Table 4, Fig. 1).

Similarly, Mollashahi et al., 2017 studied the insecticidal effect of the fruit extract of Citrullus colocynthis on Locust, Chrotogonus trachypterus. The extract was tested at different concentrations viz., 10, 20, 25, 35 and $40 \mathrm{mg} / \mathrm{l}$ on adult insects. The highest mortality $(87.50 \%)$ was recorded at a concentration of $40 \mathrm{mg} / \mathrm{l}$ and the lowest mortality (23.33\%) was observed at concentrations of $10 \mathrm{mg} / \mathrm{l}$. The mortality rate was elevated with increasing concentration and the $\mathrm{LC}_{50}$ value was estimated as 18.58 $\mathrm{mg} / \mathrm{l}$. Asiry (2015) also investigated the impact of aqueous extracts from different parts (root, stem, leaf and fruit) of bitter apple Citrullus colocynthis at five different concentrations, on $g$ the bird cherry-oat aphid, Rhopalosiphum padi (L.) (Homoptera: Aphididae) under laboratory conditions.

The stem showed a higher mortality of $R$. padi, compared to other parts, on which the mortality percentages of $R$. padi were $75 \%$, $71.25 \%, 60 \%, 43.75 \%$ and $40 \%$ respectively, when the aphids were treated with the aqueous extract of stem at 100000, 1000, 10, $0.1,0.001 \mathrm{ppm}$, respectively.

The $\mathrm{LC}_{50}$ values of stem, root, leaf and fruit of $C$. colocynthis were $0.0002,0.024,0.043$ and $0.055 \mathrm{ppm}$, respectively. Stem was revealed to be more effective than root by $0.83 \%$, leaf by $0.47 \%$ and fruit by $0.36 \%$ for controlling $R$. padi. This could be justified to a higher amount of some toxicants in the stem namely terpenoids, cucurbitacin, glycosides and flavonoids in controlling aphid pests.

Oladimeji and Kannike (2009) studied the efficacy of neem, basil leaf extracts and synthetic insecticide, lambda-cyhalothrin against okra flea beetle, Podagrica sp. The leaf extracts were applied at 5,10 and $20 \mathrm{ml}$ per litre while lambda-cyhalothrin was applied at 2.5, 3.75 and $5.0 \mathrm{ml}$ per litre and the distilled water as control.

Percentage reduction in leaf damage ranged between 21 to $43 \%$ in $O$. basilicum, 50 to 54\% in Azadirachta indica and 72 to $81 \%$ in lambda-cyhalothrin compared with the control. Haridasan et al., 2017 studied an antifeedant effect of petroleum ether and methanol leaf extracts of Vitex negundo on the stored product pest, Tribolium castaneum using a modified method of flour disc bioassay. 
Table.1 Acaricidal property of certain indigenous herbal extracts against citrus leaf mite, $P$. citri (I-Spray; Season: Summer 2018; Location: Azhagar kovil; Block: Melur, Madurai district)

\begin{tabular}{|c|c|c|c|c|c|c|}
\hline \multirow[t]{2}{*}{ Treatments } & \multicolumn{6}{|c|}{$* *$ No. of mites/ leaf } \\
\hline & $\begin{array}{l}\text { Pre-treatment } \\
\text { count }\end{array}$ & 1 DAS & 3 DAS & 7 DAS & 14 DAS & Mean \\
\hline $\mathrm{T}_{1}-$ Adathoda leaf extract @ 5\% & $\begin{array}{l}16.5 \\
(4.1)\end{array}$ & $\begin{array}{c}14.1 \\
(3.8)^{\mathrm{d}}\end{array}$ & $\begin{array}{c}12.3 \\
(3.5)^{\mathrm{d}}\end{array}$ & $\begin{array}{l}11.6 \\
(3.4)^{\mathrm{e}}\end{array}$ & $\begin{array}{c}13.4 \\
(3.7)^{\mathrm{d}}\end{array}$ & $\begin{array}{c}12.9 \\
(3.6)^{\mathrm{d}}\end{array}$ \\
\hline $\mathbf{T}_{2}-$ Citrulus fruit extract @ 5\% & $\begin{array}{l}15.9 \\
(4.0)\end{array}$ & $\begin{array}{c}9.9 \\
(3.1)^{\mathrm{b}}\end{array}$ & $\begin{array}{c}5.3 \\
(2.3)^{\mathrm{b}}\end{array}$ & $\begin{array}{c}3.5 \\
(1.9)^{\mathrm{b}}\end{array}$ & $\begin{array}{c}6.8 \\
(2.6)^{\mathrm{b}}\end{array}$ & $\begin{array}{c}6.4 \\
(2.5)^{\mathrm{b}}\end{array}$ \\
\hline $\mathbf{T}_{3}-$ Vitex leaf extract @ 5\% & $\begin{array}{l}16.5 \\
(4.1)\end{array}$ & $\begin{array}{l}11.4 \\
(3.4)^{\mathrm{bc}}\end{array}$ & $\begin{array}{l}7.8 \\
(2.8)^{\mathrm{c}}\end{array}$ & $\begin{array}{l}6.9 \\
(2.6)^{\mathrm{cd}}\end{array}$ & $\begin{array}{l}8.8 \\
(3.0)^{\mathrm{bc}}\end{array}$ & $\begin{array}{c}8.7 \\
(3.0)^{\mathrm{bc}}\end{array}$ \\
\hline $\mathrm{T}_{4}-$ Basil leaf extract @ 5\% & $\begin{array}{l}16.2 \\
(4.0)\end{array}$ & $\begin{array}{l}10.2 \\
(3.2)^{\mathrm{b}}\end{array}$ & $\begin{array}{l}6.8 \\
(2.6)^{\mathrm{bc}}\end{array}$ & $\begin{array}{l}5.7 \\
(2.4)^{\mathrm{bc}}\end{array}$ & $\begin{array}{l}8.0 \\
(2.8)^{\mathrm{b}}\end{array}$ & $\begin{array}{c}7.7 \\
(2.8)^{\mathrm{b}}\end{array}$ \\
\hline $\mathrm{T}_{5}-$ Sweet flag rhizome extract @ 5\% & $\begin{array}{l}16.4 \\
(4.0)\end{array}$ & $\begin{array}{l}13.6 \\
(3.7)^{\mathrm{cd}}\end{array}$ & $\begin{array}{c}11.4 \\
(3.4)^{\mathrm{d}}\end{array}$ & $\begin{array}{c}9.6 \\
(3.1)^{\mathrm{de}}\end{array}$ & $\begin{array}{l}10.7 \\
(3.3)^{\mathrm{cd}}\end{array}$ & $\begin{array}{c}11.3 \\
(3.4)^{\mathrm{cd}}\end{array}$ \\
\hline $\mathrm{T}_{6}$ - Propargite 50 EC @ 2ml/ lit. & $\begin{array}{l}16.2 \\
(4.0)\end{array}$ & $\begin{array}{c}6.2 \\
(2.5)^{\mathrm{a}}\end{array}$ & $\begin{array}{c}2.3 \\
(1.5)^{\mathrm{a}}\end{array}$ & $\begin{array}{c}1.6 \\
(1.3)^{\mathrm{a}}\end{array}$ & $\begin{array}{c}3.4 \\
(1.8)^{\mathrm{a}}\end{array}$ & $\begin{array}{c}3.4 \\
(1.8)^{\mathrm{a}}\end{array}$ \\
\hline $\mathbf{T}_{7}-$ Untreated check & $\begin{array}{l}16.2 \\
(4.0)\end{array}$ & $\begin{array}{c}19.6 \\
(4.4)^{\mathrm{e}}\end{array}$ & $\begin{array}{c}24.3 \\
(4.9)^{\mathrm{e}}\end{array}$ & $\begin{array}{c}27.8 \\
(5.3)^{\mathrm{f}}\end{array}$ & $\begin{array}{c}31.5 \\
(5.6)^{\mathrm{e}}\end{array}$ & $\begin{array}{c}25.8 \\
(5.1)^{\mathrm{e}}\end{array}$ \\
\hline SEd & - & 0.1682 & 0.2175 & 0.2473 & 0.1972 & 0.1984 \\
\hline$C D(p=0.05)$ & NS* & 0.3666 & 0.4739 & 0.5387 & 0.4296 & 0.4323 \\
\hline CV \% & - & 6.00 & 8.90 & 10.70 & 7.43 & 7.71 \\
\hline
\end{tabular}

*NS - Non significant; DAS - Days after spraying

**Each value is the mean of three replications @ 20 leaves/ plant drawn at random

Figures in parentheses are square root transformed values

In a column, mean denoted by common letter(s) is/are not significantly different by LSD at $\mathrm{P}=0.05 \%$

Table.2 Acaricidal property of certain indigenous herbal extracts against citrus leaf mite, $P$. citri (II-Spray; Season: Summer 2018; Location: Azhagar kovil; Block: Melur, Madurai district)

\begin{tabular}{|c|c|c|c|c|c|c|}
\hline \multirow[t]{2}{*}{ Treatments } & \multicolumn{6}{|c|}{ **No. of mites/ leaf } \\
\hline & $\begin{array}{l}\text { Pre-treatment } \\
\text { count }\end{array}$ & 1 DAS & 3 DAS & 7 DAS & 14 DAS & Mean \\
\hline $\mathrm{T}_{1}-$ Adathoda leaf extract @ 5\% & $\begin{array}{l}15.8 \\
(4.0) \\
\end{array}$ & $\begin{array}{l}12.5 \\
(3.5)^{\mathrm{c}}\end{array}$ & $\begin{array}{l}11.7 \\
(3.4)^{\mathrm{d}}\end{array}$ & $\begin{array}{l}10.2 \\
(3.2)^{\mathrm{c}}\end{array}$ & $\begin{array}{l}13.2 \\
(3.6)\end{array}$ & $\begin{array}{l}11.9 \\
(3.4)^{\mathrm{d}}\end{array}$ \\
\hline $\mathbf{T}_{2}-$ Citrulus fruit extract @ 5\% & $\begin{array}{l}15.1 \\
(3.9)\end{array}$ & $\begin{array}{l}9.5 \\
(3.1)^{\mathrm{b}}\end{array}$ & $\begin{array}{l}5.5 \\
(2.3)^{\mathrm{b}}\end{array}$ & $\begin{array}{l}3.8 \\
(1.9)^{\mathrm{a}}\end{array}$ & $\begin{array}{l}7.3 \\
(2.7)^{\mathrm{ab}}\end{array}$ & $\begin{array}{l}6.5 \\
(2.6)^{\mathrm{ab}}\end{array}$ \\
\hline $\mathbf{T}_{3}-$ Vitex leaf extract @ 5\% & $\begin{array}{l}14.9 \\
(3.9)\end{array}$ & $\begin{array}{l}11.2 \\
(3.3)^{\mathrm{bc}}\end{array}$ & $\begin{array}{l}9.1 \\
(3.0)^{\mathrm{cd}}\end{array}$ & $\begin{array}{l}7.7 \\
(2.8)^{\mathrm{bc}}\end{array}$ & $\begin{array}{l}10.7 \\
(3.3)^{\mathrm{c}}\end{array}$ & $\begin{array}{l}9.7 \\
(3.1)^{\mathrm{cd}}\end{array}$ \\
\hline $\mathbf{T}_{4}-$ Basil leaf extract @ 5\% & $\begin{array}{l}15.5 \\
(3.9)\end{array}$ & $\begin{array}{l}10.7 \\
(3.3)^{\mathrm{bc}}\end{array}$ & $\begin{array}{l}7.4 \\
(2.7)^{\mathrm{bc}}\end{array}$ & $\begin{array}{l}6.8 \\
(2.6)^{\mathrm{b}}\end{array}$ & $\begin{array}{l}9.5 \\
(3.1)^{\mathrm{bc}}\end{array}$ & $\begin{array}{l}8.6 \\
(2.9)^{\mathrm{bc}}\end{array}$ \\
\hline $\mathrm{T}_{5}-$ Sweet flag rhizome extract @ 5\% & $\begin{array}{l}15.1 \\
(3.9)\end{array}$ & $\begin{array}{l}12.8 \\
(3.6)^{\mathrm{c}}\end{array}$ & $\begin{array}{l}10.3 \\
(3.2)^{\mathrm{d}}\end{array}$ & $\begin{array}{c}9.8 \\
(3.1)^{\mathrm{c}}\end{array}$ & $\begin{array}{l}11.6 \\
(3.4)^{\mathrm{c}}\end{array}$ & $\begin{array}{l}11.1 \\
(3.3)^{\mathrm{d}}\end{array}$ \\
\hline $\mathbf{T}_{6}$ - Propargite 50 EC @ 2ml/ lit. & $\begin{array}{l}15.6 \\
(3.9)\end{array}$ & $\begin{array}{c}6.8 \\
(2.6)^{\mathrm{a}}\end{array}$ & $\begin{array}{c}3.2 \\
(1.8)^{\mathrm{a}}\end{array}$ & $\begin{array}{c}3.5 \\
(1.9)^{\mathrm{a}}\end{array}$ & $\begin{array}{c}6.4 \\
(2.5)^{\mathrm{a}}\end{array}$ & $\begin{array}{c}5.0 \\
(2.2)^{\mathrm{a}}\end{array}$ \\
\hline $\mathbf{T}_{7}-$ Untreated check & $\begin{array}{l}15.5 \\
(3.9)\end{array}$ & $\begin{array}{c}19.3 \\
(4.4)^{\mathrm{d}}\end{array}$ & $\begin{array}{l}24.5 \\
(4.9)^{\mathrm{e}}\end{array}$ & $\begin{array}{c}28.4 \\
(5.3)^{\mathrm{d}}\end{array}$ & $\begin{array}{l}31.7 \\
(5.6)^{\mathrm{e}}\end{array}$ & $\begin{array}{l}26.0 \\
(5.1)^{\mathrm{e}}\end{array}$ \\
\hline SEd & - & 0.1673 & 0.2034 & 0.2098 & 0.1737 & 0.1847 \\
\hline$C D(p=0.05)$ & NS* & 0.3645 & 0.4431 & 0.4572 & 0.3785 & 0.4024 \\
\hline CV \% & - & 6.03 & 8.15 & 8.65 & 6.15 & 6.98 \\
\hline
\end{tabular}

*NS - Non significant; DAS - Days after spraying

**Each value is the mean of three replications @ 20 leaves/ plant drawn at random

Figures in parentheses are square root transformed values

In a column, means denoted by common letter(s) is/are not significantly different by LSD at $\mathrm{P}=0.05 \%$ 
Table.3 Acaricidal property of certain indigenous herbal extracts against citrus leaf mite, $P$. citri (III-Spray; Season: Summer 2018; Location: Azhagar kovil; Block: Melur, Madurai district)

\begin{tabular}{|c|c|c|c|c|c|c|}
\hline \multirow[t]{2}{*}{ Treatments } & \multicolumn{6}{|c|}{$* *$ No. of mites/ leaf } \\
\hline & $\begin{array}{c}\text { Pre- } \\
\text { treatment } \\
\text { count }\end{array}$ & 1 DAS & 3 DAS & 7 DAS & 14 DAS & Mean \\
\hline $\mathbf{T}_{1}-$ Adathoda leaf extract @ 5\% & $\begin{array}{c}14 \\
(3.7)\end{array}$ & $\begin{array}{c}13 \\
(3.6)^{\mathrm{de}}\end{array}$ & $\begin{array}{c}12.6 \\
(3.5)^{\mathrm{d}}\end{array}$ & $\begin{array}{c}11 \\
(3.3)^{\mathrm{c}}\end{array}$ & $\begin{array}{c}14.1 \\
(3.8)^{\mathrm{b}}\end{array}$ & $\begin{array}{l}12.70 \\
(3.6)^{\mathrm{c}}\end{array}$ \\
\hline $\mathbf{T}_{2}-$ Citrulus fruit extract @ 5\% & $\begin{array}{l}14.8 \\
(3.8)\end{array}$ & $\begin{array}{l}8.4 \\
(2.9)^{\mathrm{b}}\end{array}$ & $\begin{array}{c}4.4 \\
(2.1)^{\mathrm{a}}\end{array}$ & $\begin{array}{c}3.2 \\
(1.8)^{\mathrm{a}}\end{array}$ & $\begin{array}{c}7.8 \\
(2.8)^{\mathrm{a}}\end{array}$ & $\begin{array}{c}6.0 \\
(2.4)^{\mathrm{ab}}\end{array}$ \\
\hline$T_{3}-$ Vitex leaf extract @ 5\% & $\begin{array}{l}14.1 \\
(3.8)\end{array}$ & $\begin{array}{l}11.5 \\
(3.4)^{\mathrm{cd}}\end{array}$ & $\begin{array}{c}9.4 \\
(3.1)^{\mathrm{c}}\end{array}$ & $\begin{array}{c}8.6 \\
(2.9)^{\mathrm{c}}\end{array}$ & $\begin{array}{c}11.5 \\
(3.4)^{\mathrm{b}}\end{array}$ & $\begin{array}{c}10.3 \\
(3.2)^{\mathrm{c}}\end{array}$ \\
\hline $\mathrm{T}_{4}-$ Basil leaf extract @ 5\% & $\begin{array}{l}13.9 \\
(3.7)\end{array}$ & $\begin{array}{l}9.6 \\
(3.1)^{\mathrm{bc}}\end{array}$ & $\begin{array}{l}6.8 \\
(2.6)^{\mathrm{b}}\end{array}$ & $\begin{array}{c}6.1 \\
(2.5)^{\mathrm{b}}\end{array}$ & $\begin{array}{c}8.8 \\
(3.0)^{\mathrm{a}}\end{array}$ & $\begin{array}{l}7.8 \\
(2.8)^{\mathrm{b}}\end{array}$ \\
\hline $\mathbf{T}_{5}-$ Sweet flag rhizome extract @ 5\% & $\begin{array}{l}14.2 \\
(3.8)\end{array}$ & $\begin{array}{l}13.6 \\
(3.7)^{\mathrm{de}}\end{array}$ & $\begin{array}{c}12.4 \\
(3.5)^{\mathrm{d}}\end{array}$ & $\begin{array}{l}11.3 \\
(3.4)^{\mathrm{c}}\end{array}$ & $\begin{array}{l}13.7 \\
(3.7)^{\mathrm{b}}\end{array}$ & $\begin{array}{c}12.8 \\
(3.6)^{\mathrm{c}}\end{array}$ \\
\hline $\mathbf{T}_{6}-$ Propargite 50 EC @ 2ml/ lit. & $\begin{array}{l}14.5 \\
(3.8)\end{array}$ & $\begin{array}{c}5.3 \\
(2.3)^{\mathrm{a}}\end{array}$ & $\begin{array}{c}3.8 \\
(1.9)^{\mathrm{a}}\end{array}$ & $\begin{array}{c}4.7 \\
(2.2)^{\mathrm{ab}}\end{array}$ & $\begin{array}{c}6.8 \\
(2.6)^{\mathrm{a}}\end{array}$ & $\begin{array}{c}5.2 \\
(2.3)^{\mathrm{a}}\end{array}$ \\
\hline $\mathbf{T}_{7}-$ Untreated check & $\begin{array}{l}13.9 \\
(3.7)\end{array}$ & $\begin{array}{c}14.7 \\
(3.8)^{\mathrm{e}}\end{array}$ & $\begin{array}{c}19.2 \\
(4.4)^{\mathrm{e}}\end{array}$ & $\begin{array}{c}27.6 \\
(5.3)^{d}\end{array}$ & $\begin{array}{c}32.4 \\
(5.7)^{\mathrm{c}}\end{array}$ & $\begin{array}{c}23.5 \\
(4.8)^{\mathrm{d}}\end{array}$ \\
\hline SEd & - & 0.1747 & 0.1996 & 0.2041 & 0.1705 & 0.1842 \\
\hline$C D(p=0.05)$ & NS* & 0.3806 & 0.4349 & 0.4447 & 0.3714 & 0.4013 \\
\hline CV \% & - & 6.57 & 8.10 & 8.24 & 5.87 & 6.97 \\
\hline
\end{tabular}

*NS - Non significant; DAS - Days after spraying

**Each value is the mean of three replications @ 20 leaves/ plant drawn at random

Figures in parentheses are square root transformed values

In a column, means denoted by common letter(s) is/are not significantly different by LSD at $\mathrm{P}=0.05 \%$

Table.4 Acaricidal property of certain indigenous herbal extracts against citrus leaf mite, $P$. citri (Season: Summer 2018; Location: Azhagar kovil; Block: Melur, Madurai district)

\begin{tabular}{|c|c|c|c|c|c|c|c|}
\hline \multirow[t]{2}{*}{ Treatments } & \multicolumn{4}{|c|}{ *No. of mites/ leaf } & \multirow[b]{2}{*}{$\begin{array}{c}\% \\
\text { reduction } \\
\text { over } \\
\text { untreated } \\
\text { check }\end{array}$} & \multirow[t]{2}{*}{ Yield (t/ha) } & \multirow{2}{*}{$\begin{array}{c}\text { Cost } \\
\text { benefit } \\
\text { ratio }\end{array}$} \\
\hline & I Spray & II Spray & $\begin{array}{l}\text { III } \\
\text { Spray }\end{array}$ & $\begin{array}{l}\text { Cumulative } \\
\text { Mean }\end{array}$ & & & \\
\hline $\mathrm{T}_{1}$ - Adathoda leaf extract @ 5\% & $\begin{array}{l}12.9 \\
(3.6)^{\mathrm{d}}\end{array}$ & $\begin{array}{l}11.9 \\
(3.4)^{\mathrm{d}}\end{array}$ & $\begin{array}{l}12.70 \\
(3.6)^{\mathrm{c}}\end{array}$ & $\begin{array}{l}12.50 \\
(3.5)^{\mathrm{e}}\end{array}$ & 50.19 & $\begin{array}{l}5.90 \\
(0.8)^{\mathrm{cd}}\end{array}$ & 1: 1.4 \\
\hline $\mathbf{T}_{2}-$ Citrulus fruit extract @ 5\% & $\begin{array}{c}6.4 \\
(2.5)^{\mathrm{b}}\end{array}$ & $\begin{array}{c}6.5 \\
(2.6)^{\mathrm{ab}}\end{array}$ & $\begin{array}{c}6.0 \\
(2.4)^{\mathrm{ab}}\end{array}$ & $\begin{array}{c}6.30 \\
(2.5)^{\mathrm{ab}}\end{array}$ & 74.90 & $\begin{array}{c}9.60 \\
(1.0)^{\mathrm{a}}\end{array}$ & $1: 2.2$ \\
\hline $\mathbf{T}_{3}-$ Vitex leaf extract @ 5\% & $\begin{array}{l}8.7 \\
(3.0)^{\mathrm{bc}}\end{array}$ & $\begin{array}{l}9.7 \\
(3.1)^{\mathrm{cd}}\end{array}$ & $\begin{array}{l}10.3 \\
(3.2)^{\mathrm{c}}\end{array}$ & $\begin{array}{l}9.60 \\
(3.1)^{\mathrm{cd}}\end{array}$ & 61.75 & $\begin{array}{l}8.10 \\
(0.9)^{\mathrm{abc}}\end{array}$ & $1: 1.9$ \\
\hline $\mathbf{T}_{4}-$ Basil leaf extract @ 5\% & $\begin{array}{l}7.7 \\
(2.8)^{\mathrm{b}}\end{array}$ & $\begin{array}{l}8.6 \\
(2.9)^{\mathrm{bc}}\end{array}$ & $\begin{array}{c}7.8 \\
(2.8)^{\mathrm{b}}\end{array}$ & $\begin{array}{l}8.00 \\
(2.8)^{\mathrm{bc}}\end{array}$ & 68.12 & $\begin{array}{l}8.50 \\
(0.9)^{\mathrm{ab}}\end{array}$ & $1: 2.0$ \\
\hline $\mathbf{T}_{5}-$ Sweet flag rhizome extract @ 5\% & $\begin{array}{l}11.3 \\
(3.4)^{\mathrm{cd}}\end{array}$ & $\begin{array}{c}11.1 \\
(3.3)^{\mathrm{d}}\end{array}$ & $\begin{array}{l}12.8 \\
(3.6)^{\mathrm{c}}\end{array}$ & $\begin{array}{l}11.70 \\
(3.4)^{\mathrm{de}}\end{array}$ & 53.38 & $\begin{array}{l}6.40 \\
(0.8)^{\mathrm{bc}}\end{array}$ & $1: 1.5$ \\
\hline $\mathrm{T}_{6}$ - Propargite 50 EC @ 2ml/ lit. & $\begin{array}{c}3.4 \\
(1.8)^{\mathrm{a}}\end{array}$ & $\begin{array}{l}5.0 \\
(2.2)^{\mathrm{a}}\end{array}$ & $\begin{array}{c}5.2 \\
(2.3)^{\mathrm{a}}\end{array}$ & $\begin{array}{l}4.50 \\
(2.1)^{\mathrm{a}}\end{array}$ & 82.07 & $\begin{array}{l}11.20 \\
(1.0)^{\mathrm{a}}\end{array}$ & $1: 2.6$ \\
\hline $\mathbf{T}_{7}-$ Untreated check & $\begin{array}{l}25.8 \\
(5.1)^{\mathrm{e}}\end{array}$ & $\begin{array}{l}26.0 \\
(5.1)^{\mathrm{e}}\end{array}$ & $\begin{array}{l}23.5 \\
(4.8)^{\mathrm{d}}\end{array}$ & $\begin{array}{l}25.10 \\
(5.0)^{\mathrm{f}}\end{array}$ & - & $\begin{array}{l}4.30 \\
(0.6)^{\mathrm{d}}\end{array}$ & - \\
\hline SEd & 0.1984 & 0.1847 & 0.1842 & 0.1882 & - & 0.068 & - \\
\hline$C D(p=0.05)$ & 0.4323 & 0.4024 & 0.4013 & 0.4101 & - & 0.148 & - \\
\hline CV \% & 7.71 & 6.98 & 6.97 & 7.18 & - & 9.64 & - \\
\hline
\end{tabular}

*Each value is the mean of three replications @ 20 leaves/ plant drawn at random

Figures in parentheses are square root transformed values

For yield data log transformation is adapted

In a column, means denoted by common letter(s) is/are not significantly different by LSD at $\mathrm{P}=0.05 \%$

The current market rate of Rs. $30 / \mathrm{kg}$ is considered to work out the CBR 


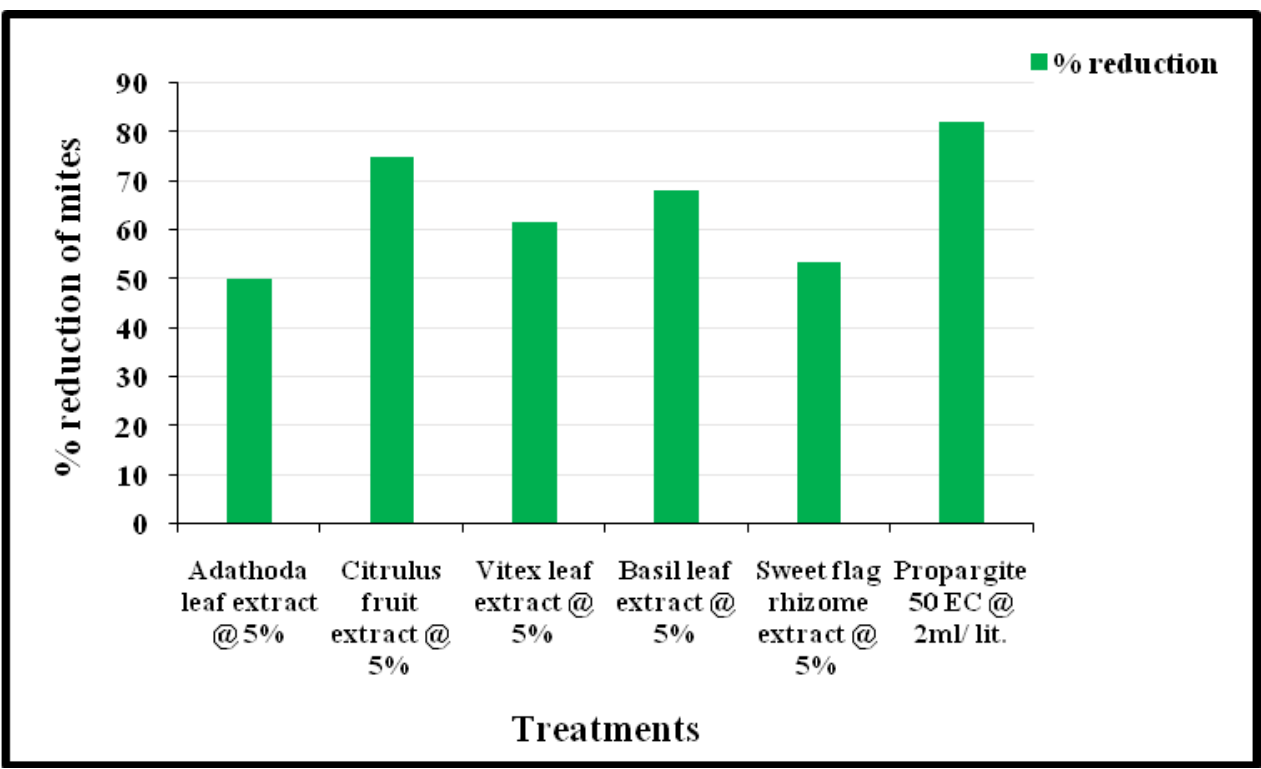

Fig.1 Acaricidal property of certain indigenous herbal extracts against citrus leaf mite, $P$. citri

The insects were allowed to feed on wheat flour treated with various concentrations $(1.25 \%, 2.5 \%$ and $5 \%)$ of petroleum ether and methanol extracts of $V$. negundo for $24 \mathrm{hrs}$ in 'no choice' chambers. Both the petroleum ether and methanol extracts were significantly affected the growth, consumption and utilization of food and caused antifeedant effect in $T$. castaneum. The reductions in relative growth rate $(\mathrm{RGR})(\mathrm{P}=0.37)$ and relative food consumption rate (RCR) $(\mathrm{P}<0.05)$ were more prominent for $2.5 \%$ and $5 \%$ of both the extracts.

Among the herbal extracts evaluated for their toxicity at field conditions against citrus leaf mite $P$. citri revealed Citrulus fruit extract, basil leaf extract and Vitex leaf extract @5 percent are found promising, occupying the top three ranks with the highest per cent reduction of mites viz., 74.90, 68.12 and 61.75 , respectively. This has reflected on increased fruit yield and CBR. Thus these herbal extracts are very effective against citrus mite besides being cheaper and safer to the environment at recommended doses. This may be promoted in future as ecofriendly strategy, which ofcourse warrants further indepth studies to understand the exact active principle and mode of action, its effect on the non-target entomophages besides residual toxicity in fruits recommending as an alternative to synthetic pesticides.

\section{References}

Asiry, K.A. 2015. Aphidicidal activity of different aqueous extracts of bitter apple, Citrullus colocynthis (L.) against the bird cherry-oat aphid, Rhopalosiphum padi (L.) (Homoptera: Aphididae) under laboratory conditions. The Journal of Animal and Plant Sciences, 25(1): 456-462.

Butani, D.K. 1979. Insect pests of citrus and their control. Pesticides, 13(4): 27-33.

Dhooria, M.S. and Gupta, S.K. 1998. Observation on phytophagous and predaceous mites found on citrus from Punjab State. Abstract No. 2066. In National Seminar on Entomology in $21^{\text {st }}$ century, held at Udaipur, during April 30-May 2, 1998.

Dhooria, M.S., M.B. Bhullar and Mallik, B. 2005. Mite pests of citrus and their management in India. $28 \mathrm{pp}$. AINP (Agricultural Acarology), UAS, Bangalore. 
Haridasan, P., M. Gokuldas and Ajaykumar, A.P. 2017. Antifeedant effects of vitex negundo leaf extracts on the stored product pest, Tribolium castaneum (Coleoptera: Tenebrionidae). International Journal of Pharmacy and Pharmaceutical Sciences, 9(3): 18-22.

Kranz, J., H. Schmutterer and Koch, W. 1977. Diseases, pests and weeds in tropical crops. Paul Parey, Berlin, Germany.

Leclerg, E.L. 1965. Losses in Agriculture. U.S. Dept. Agr., A.R.S. Handbook no. 291, 120 pp.

Mollashahi, H., A.Mirshekari, M. Ghorbani and Tarrah, A. 2017. Insecticidal effect of the fruit extract bitter melon (Citrullus colocynthis) on Locust, Chrotogonus trachypterus (Orth: Pyrgomorphidae). Biosciences Biotechnology Research Asia, 14(4): 1285-1289.

Oladimeji, A. and Kannike, M.A. 2010. Comparative studies on the efficacy of neem, basil leaf extracts and synthetic insecticide, lambda-cyhalothrin, against Podagrica spp. on okra. African Journal of Microbiology Research, 4(1): 033-037.

Poovizhiraja, B., C. Chinniah, M. Murugan, S. Irulandi, K.E.A. Aiyanathan and Balamohan,T.N. 2019. Impact of weather parameters on the population dynamics of major sucking pests of acid lime, Citrus aurantifolia Swingle. Journal of Entomology and Zoology Studies, 7(3): 197-201.

Prasad, V. 1974. A catalogue of mites of India. Indira Acarology Publishing House, Ludhiana (Punjab) India 320 pp.

Rao, A.R., P.K. Rao and Jyotsna, K.P. 2015. Efficacy of certain natural insecticides against Citrus leaf miner, Phyllocnistis citrella Stainton as prophylactic and curative measures on Sathgudi Sweet Orange. Pest Management in Horticultural Ecosystems, 21(1): 1115.

\section{How to cite this article:}

Chinniah, C., K. Naveena and Shanthi, M. 2020. Acaricidal Property of Certain Indigenous Herbal Extracts against Citrus Leaf Mite, Panonychus citri on Acid Lime. Int.J.Curr.Microbiol.App.Sci. 9(05): 2443-2449. doi: https://doi.org/10.20546/ijcmas.2020.905.280 